\title{
ARTRODESE NA COLUNA CERVICAL UTILIZANDO SICAP COMO SUBSTITUTO DE ENXERTO ÓSSEO
}

\author{
CERVICAL SPINE FUSION UTILIZING SILICATED CALCIUM PHOSPHATE BONE GRAFT \\ SUBSTITUTE (SICAP) \\ ARTRODESIS EN LA COLUMNA CERVICAL UTILIZANDO SICAP COMO SUSTITUTO DE \\ INJERTO ÓSEO
}

Juliano Fratezl ${ }^{1}$, Harry Gebhard ${ }^{1}$, Roger HärtL ${ }^{3}$

\begin{abstract}
RESUMO
Objetivo: Substitutos de enxerto ósseo autólogo foram desenvolvidos para evitar as complicações da retirada de enxerto ósseo autólogo. SiCaP (Actifuse, ApaTech EUA, Reino Unido) é um enxerto ósseo composto de cálcio-fosfato com um substituição de silicato na estrutura química, com uma estrutura tridimensional que parece osso natural. Métodos: 19 pacientes foram submetidos à fusão óssea cervical e analisados retrospectivamente. A avaliação radiográfica e avaliação clínica foram realizadas utilizando o questionário Neck Disability Index e a escala análoga da dor (VAS) pré- e pós-operação. Resultados: O período médio de acompanhamento pós-operatório foi de 14 meses \pm 5 meses (7-30 meses). 11 pacientes foram submetidos à fusão via anterior; 5 pacientes via posterior e 3 pacientes via anterior e posterior. A revisão radiográfica mostrou 19/19 (100\%) de fusão óssea, nenhum caso apresentou subsidência, quebra ou soltura de material de implante ou movimento nos níveis fusionados. Nenhum exemplo de ossificação heterotópica ou de crescimento ósseo intracanal foi observado. Clinicamente, os escores médios do Neck Disability decresceram 13,3 pontos (media pré-op. de 34,5, pós-op. de 21,2, melhora de 39\%), a média da VAS para dor cervical decresceu 2 pontos (2,7 pré-op para 0,7 pós-op.; melhora de 74,1\%). Não foram observadas complicações como infecção, osteólise ou edema excessivo das partes moles. Conclusão: Os resultados preliminares obtidos nesta série foram encorajadores com o uso do SICaP como enxerto ósseo, com sólida fusão óssea obtida em todos os casos e sem formação de ossificação heterotópica ou crescimento de osso intracanal. SlcaP demonstra ser um substituto confiável para o enxerto ósseo autólogo na coluna cervical.
\end{abstract}

Descritores: Coluna vertebral; Transplantes ósseos; Substitutos ósseos; Fusão vertebral; Cerâmica.

\begin{abstract}
Objective: Bone graft substitutes have been developed to obviate the need for autograft from the iliac crest and its resultant complications. SiCaP (Actifuse, ApaTech US, UK) is a calcium phosphate bone graft substitute with selective controlled silicate substitution in a patented 3-dimensional structure resembling natural bone. Methods: 19 patients who underwent cervical spine fusion were retrospectively reviewed. Radiographic evaluation and clinical evaluation were performed using Neck Disability Index questionnaire and Visual Analog Scale (VAS) pre- and post-operatively. Results: The mean post-operative follow-up was $14 \pm 5$ months, range 7-30 months. Eleven patients had an anterior approach, five patients had a posterior approach, and 3 had combined anterior-posterior approaches. Radiographic review showed 19/19 (100\%) patients were considered fused, with no subsidence, hardware breakage, or hardware loosening. No instances of heterotopic bone formation or intracanal boney ingrowths were observed. Clinically, average Neck Disability scores decreased 13.3 points (pre-op 34.5, post-op 21.2, a 39\% improvement); average VAS neck pain scores decreased 2.2 points (4.9 pre-op to 1.9 post-op; a $44.9 \%$ improvement; average VAS arm pain decreased 2.0 points (2.7 pre-op to 0.7 post-op, a $74.1 \%$ improvement). There were no complications such as infection, osteolysis, or abnormal swelling of soft tissues. Conclusions: Preliminary results from this series with the use of SiCaP bone graft substitute were encouraging, with solid fusion occurring in all subjects, and no heterotopic bone formation or intracanal bone ingrowths. SiCaP seems to be a reliable alternative to autograft on cervical spine fusion achieving solid fusion with no complications.
\end{abstract}

Keywords: ACDF; Posterior cervical fusion; Bone graft substitute.

\section{RESUMEN}

Objetivo: Sustitutos de injerto óseo autólogo fueron desarrollados para evitar las complicaciones de la recogida de injerto óseo autólogo. $\mathrm{SiCaP}$ (Actifuse, ApaTech EE.UU, Reino Unido) es un injerto óseo compuesto de calcio-fosfato con una sustitución de silicato en la estructura química, con una estructura tridimensional que parece hueso natural. Métodos: 19 pacientes fueron sometidos a fusión ósea cervical y analizados retrospectivamente. La evaluación radiográfica y la evaluación clínica fueron realizadas utilizandose el cuestionario Neck Disability Index y la escala análoga del dolor (VAS) pre y postoperación. Resultados: El período promedio de seguimiento postoperatorio fue de 14 meses \pm 5 meses (7-30 meses). Once pacientes fueron sometidos a fusión vía anterior; 5 pacientes vía posterior y 3 pacientes vía anterior y posterior. La revisión radiográfica mostró 19/19 (100\%) de fusión ósea, ningún caso presentó subsidencia, rotura o soltura de material de implante o movimiento en los niveles fusionados. Ningún ejemplo de osificación heterotópica o de crecimiento óseo intracanal fue observado. Clínicamente, el promedio de las puntuaciones del Neck Disability disminuyeron 13,3 puntos (promedio preop. de 34,5, postop. de 21,2, mejora de 39\%), el promedio de VAS para dolor cervical disminuyó 2 puntos (2,7 preop. para 0,7 postop.; mejora de $74,1 \%)$. No fueron observadas complicaciones como infección, osteólisis o edema excesivo de las partes blandas. Conclusión:

\footnotetext{
1 - Médico Estagiário em Cirurgia da Coluna - NewYork Weill Cornell Medical Center - NewYork - NY. U.S.

2 - Médico Estagiário em Cirurgia da Coluna - New York Weill Cornell Medical Center - New York - NY. U.S.

3 - Chefe do Departamento de Cirurgia da Coluna - New York Weill Cornell MedicalCenter - New York - NY. U.S.
}

Trabalho realizado na NewYork Weill Cornell Medical - New York - NY. U.S.

Correspondência: Rua Dr. Pedro Soares de Camargo, 97, apto 1402, Anhangabau. Jundiai - SP. Brasil. 13208-080 - E-mail: fratezi@yahoo.com.br

Recebido em 30/06/2010, aceito em 30/03/2011 
Los resultados preliminares obtenidos en esta serie feuron estimulantes con el uso de SICaP como injerto óseo, con sólida fusión ósea obtenida en todos los casos y sin formación de osificación heterotópica o crecimiento de hueso intracanal. SlcaP demuestra ser un sustituto confiable para el injerto óseo autólogo en la columna cervical.

Descriptores: Columna vertebral; Trasplantes óseos; Sustitutos óseos; Fusión vertebral; Cerámica.

\section{INTRODUÇÃO}

Fusão de segmentos da coluna cervical continua um procedimento comum para tratamento de diversas doenças da coluna, como: espondilólise, a causa não-traumática mais comum de dor cervical, acompanhada ou não de neuropatia ${ }^{1}$. Alterações degenerativas como, hérnia de disco, degeneração discal, osteófitos e hipertrofia dos ligamentos, podem causar compressão com dor cervical, radiculopatia e mielopatia ${ }^{2}$. Fusão cervical também pode ser utilizada no tratamento de fraturas instáveis, tumores e alguns casos de osteomielite ${ }^{3,4}$.

Enxerto autólogo da crista ilíaca é ainda muito utilizado, tem altas taxas de fusão óssea, porem é um método que apresenta complicações como: estoque limitado, necessidade de outra incisão, aumento do tempo operatório, sangramento, e possibilidade de dor persistente no sítio doador; para evitar essas complicações, vários substitutos sintéticos de enxerto ósseo vem sendo desenvolvidos ${ }^{5,6}$.

Neste estudo foi utilizado um substituto de enxerto composto silicato cácio fosfato, até o presente momento não há outras publicações sobre o uso deste tipo de enxerto em cirurgias de fusão cervical tanto em vias anteriores quanto posteriores. Não existem outras publicações na literatura sobre fusão cervical utilizando SlcaP como enxerto.

\section{MATERIAS E MÉTODOS}

Foi obtido aprovação da comissão de ética (IRB) do New york Weill Cornell Medical Hospital. Um total de 19 pacientes com media de idade de 51 anos (22-88 anos) foram submetidos à fusão cervical entre outubro de 2007 e dezembro de 2008 foram revisados os dados clínicos e radiológicos.

A via anterior, com ou sem corpectomia de um nível, foi indicada nos casos nos quais a causa da compressão era anterior, incluindo hérnia de disco, doença degenerativa discal, formações osteofitárias e diminuição da lordose. A via posterior utilizada nos casos nos quais a causa da compressão eram elementos posteriores, como hipertrofia ligamentar. Via combinada posterior e anterior foi utilizada apenas em dois casos: uma fratura instável de C6 com deformidade em cifose que necessitava de correção; o Segundo caso foi uma revisão de uma discectomia e fusão anterior e correção deformidade em cifose.

Análise radiográfica consistiu de radiografias pré-op, três, seis e um ano após cirurgia ou na última visita pós-op. foi considerado como fusão presença de ponte óssea entre os segmentos no TC, ausência de subsidence, soltura, quebra ou movimento do material de implante nas imagens radiográficas dinâmicas.

Análise clínica foi feita pelo questionário Neck Disability Index e Visual Anologue Scale (VAS) pré-op e na última visita post-op.

\section{TÉCNICA CIRÚRGICA}

Via anterior: exposição, discectomia e descompressão foram feitas por um incisão do lado direito, como descrita por Smith-robinson ${ }^{7}$. As placas terminais foram cuidadosamente preparadas e ligamento longital posterior removido. Foi utilizado cage de PEEK preenchido com SICap. Em oito casos foi feito suplementação com placa anterior e em três casos foi utilizado cage Zero-plate (syntes Co. Inc. Olothurn, Switzerland), o qual possui fixação com parafusos no próprio cage, dispensando uso de placa anterior.
Via Posterior: paciente posicionado decúbito ventral com a cabeça em um apoio de Mayfield (Ohio Medical Instrument Co., Inc, Cincinnati, $\mathrm{OH}$ ). Incisão posterior na linha media e cuidadosa exposicão das laminas e das massas laterais; feito laminectomia descompressiva, retirada do ligamento amarelo, fascetectomia medial e foraminotomias; após, feito descompressão, instrumentação com parafusos de massa lateral e barras colocados com auxilio de neuronavegação (Brainlab Co. Inc. Feldkirchen, Germany) e fluoroscopia.

\section{RESULTADOS}

O tempo médio de acompanhamento pós-operatório foi de 14 \pm 5 meses(variação de 7-30). Em 11 pacientes foi feito via anterior, cinco pacientes via posterior e em três pacientes via combinada anterior e posterior. Apenas três pacientes são tabagistas, diagnóstico e tipo de instrumentação mostrado na Tabela 1.

Revisão radiográfica mostrou 19/19 (100\%) dos pacientes foram considerados fusionados, não foi observado nenhum caso de crescimento de osso heterotópico intra-canal. A média do Neck Disability score cervical decresceu 13.3 pontos (pré-op $34.5 \pm 8$, pós-op $21.2 \pm 6$, melhora de 39\%). A média do VAS para dor cervical decresceu 2.2 pontos. ( $4.9 \pm 1$ pre-op to $1.9 \pm .7$ pós-op; melhora de $44.9 \%$ ). A media para VAS para dor nos membros superiors decresceu 2.0 pontos ( $2.7 \pm 1$ pre-op to $0.7 \pm .5$ pós-op, melhora de $74.1 \%)$. Não foram observadas complicacões como: osteolise ou edema anormal de partes moles.

Tabela 1. Descrição de diagnóstico, viade acesso e material de implante.

\begin{tabular}{c|c|c|c}
\hline Paciente & Diagnóstico & Via & Material de implante \\
\hline 1 & Hérnia de disco & Anterior & PEEK Cage/ Placa Cervical \\
\hline 2 & Hérnia de disco & Anterior & PEEK Cage/ Placa Cervical \\
\hline 3 & Doença degenerativa discal & Anterior & PEEK Cage/ Placa Cervical \\
\hline 4 & Mielopatia & Anterior & PEEK Cage/ Placa Cervical \\
\hline 5 & Mielopatia & Anterior & PEEK Cage/ Placa Cervical \\
\hline 6 & Hérnia de disco & Anterior & PEEK Cage/ Placa Cervical \\
\hline 7 & Hérnia de disco & Anterior & PEEK Cage/ Placa Cervical \\
\hline 8 & Fratura de C3-C4 & Anterior & PPEK Cage/Cervical Plate \\
\hline 9 & Hérnia de disco & Anterior & Zero Plate \\
\hline 10 & Hérnia de disco & Anterior & Zero Plate \\
\hline 11 & Hérnia de disco & Anterior & Zero Plate \\
\hline 12 & C2 Pannus, mielopatia & Posterior & Parafusos de massa lateral \\
\hline 13 & DISH & Posterior & Parafusos de massa lateral \\
\hline 14 & Hérnia de disco & Posterior & Parafusos de massa lateral \\
\hline 15 & Hérnia de disco & Posterior & Parafusos de massa lateral \\
\hline 16 & Fratura facetaria & Posterior & Parafusos de massa lateral \\
\hline 17 & Revisão de artrodese, & Anterior/Posterior & $\begin{array}{l}\text { PEEK Cage/placa Cervical/ } \\
\text { Parafusos de massa lateral }\end{array}$ \\
\hline 19 & Fratura de C6 & Anterior/Posterior & $\begin{array}{l}\text { PEEK Cage/placa Cervical/ } \\
\text { Parafusos de massa lateral }\end{array}$ \\
\hline 19 & Pannus odontóide, & Anterior/Posterior & $\begin{array}{l}\text { PEEK Cage/placa Cervical/ } \\
\text { Parafusos de massa lateral }\end{array}$ \\
\hline 10 itia & &
\end{tabular}




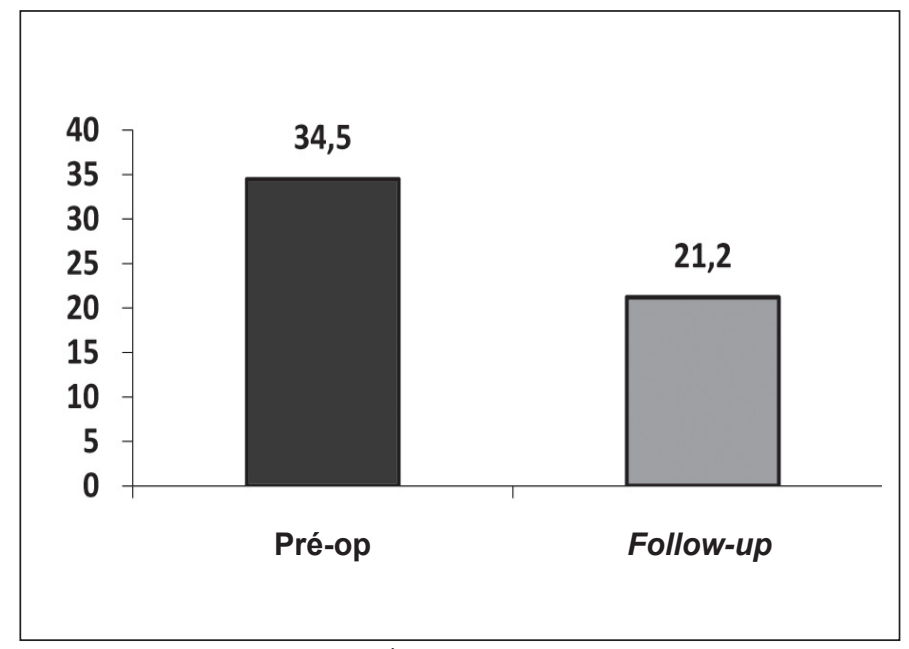

Figure 1. Neck disability index (Índice de Incapacidade Cervical).

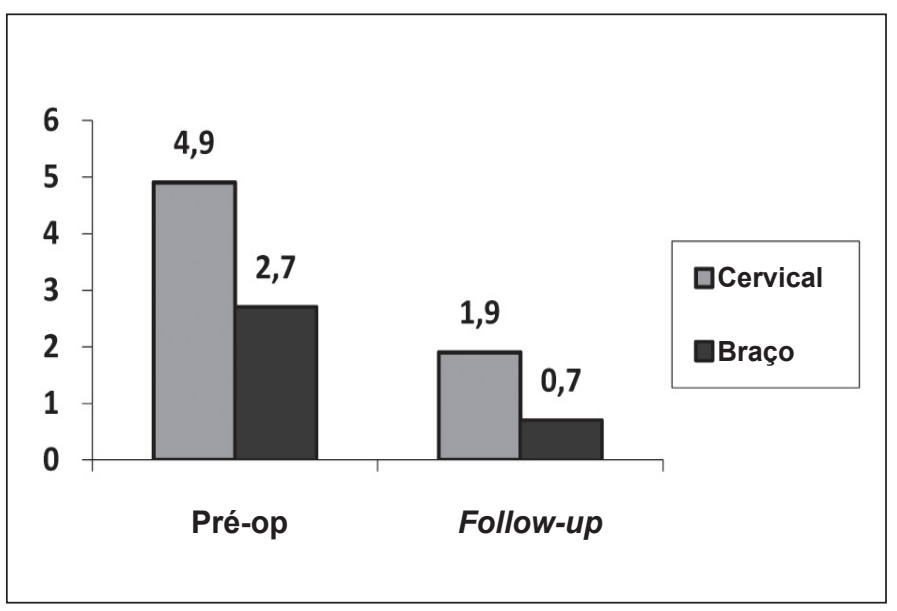

Figure 2. VAS (Escala Analógica Visual).

\section{DISCUSSÃO}

Enxerto autólogo é historicamente considerado o "padrão-ouro" para fusão óssea, porém as complicações inerentes ao método estão levando ao desenvolvimento de alternativas para substituto do enxerto autólogo ${ }^{5}$. Existem três principais tipos de substitutos: matriz óssea desmineralizada (DBM). Proteína Morfogênica óssea
(BMP) e as cerâmicas, como hidroxiapatita $(\mathrm{HA})$, tricálcio-fosfatos (CaP e o silicato cálcio fosfato (SiCaP-Actifuse $)^{5,10-13}$, sendo o ultimo utilizado no presente estudo.

Taxas de fusão óssea utilizando enxerto de cadáveres são muito variáveis, dependendo da fonte, qualidade do osso e ainda do método de esterilização. Métodos de estelização modernos diminuíram muito o risco de transmissão de doenças por este tipo de enxerto ${ }^{8.9}$. porém ainda existe o risco, levando muitos cirurgiões e pesquisadores a desenvolver alternativas sintéticas de enxerto ósseo,

Matriz Desmiralizada Óssea tem altas taxas de fusão óssea em alguns estudos, porem baixas taxas também foram publicadas em outras séries ${ }^{16-20}$. proteína Morfogenética Óssea (BMP), tem altas taxas de fusão em vários estudos, porém, o custo elevado, e complicações relacionadas ao uso deste produto, particularmente em estudos recentes de complicações em cirurgia de descompressão anterior e fusão na coluna cervical, tidas como: crescimento de osso além dos limites do espaço discal, inclusive para parte intracanal com ou sem compressão sobre estruturas nervosas, edema anormal de partes moles e osteólise de corpos vertebrais, levando a falha da cirurgia. ${ }^{21-25}$. Enxertos cerâmicos são a mais recende opção de substituto sintético de enxerto ósseo, têm demonstrado ser seguro, mas com taxas de fusão variáveis, dependendo principalmente da composição química da cerâmica. ${ }^{26-29}$. o processo químico do SlcaP (Actifuse), inclui a criação de uma estrutura tridimensional perto dos moldes do osso humano em sua estrutura microscópica e a adição de silicato em uma taxa de $0.8 \%$ do peso do enxerto.

Traços de silicato são detectáveis em osso imaturo em desenvolvimento como parte do processo natural desenvolvimento ósseo. Ele também proporciona ligação entre as moléculas de colágeno e uma regulação para cima da proliferação e diferenciação de osteoblastos. A substituição seletiva do íon de silicato na matriz de cálcio-fosfato, promove mudanças na superfície química do material, o que aumenta o bioatividade do material, promovendo maior ligação de proteínas envolvidas no processo de osteogênese. ${ }^{30-36}$.

\section{CONCLUSÃO}

Resultados preliminares desta série com o uso de SiCaP como substituto de enxerto sintético na coluna cervical são encorajadores; com fusão sólida sendo demonstrada em todos os 19 casos, sem formação de osso heterotópico. SiCaP se mostrou uma alternativa segura para substituto do enxerto autólogo, porém necessita de mais estudos prospectivos sobre o tema.

\section{REFERÊNCIAS}

1. Uchida K, Nakajima H, Sato R, Yayama T, Mwaka ES, Kobayashi S, et al. Cervical spondylotic myelopathy associated with kyphosis or sagittal sigmoid alignment: outcome after anterior or posterior decompression. J Neurosurg Spine. 2009;11(5):521-8.

2. Fehlings $M G$, Arvin B. Surgical management of cervical degenerative disease: the evidence related to indications, impact, and outcome. J Neurosurg Spine. 2009;11(2):97-100.

3. Lu DC, Wang V, Chou D. The use of allograft or autograft and expandable titanium cages for the treatment of vertebral osteomyelitis. Neurosurgery. 2009;64(1):122-9.

4. Cloyd JM, Acosta FL Jr, Ames CP. Effect of age on the perioperative and radiographic complications of multilevel cervicothoracic spinal fusions. Spine (Phila Pa 1976). 2008;33(26):E977-82.

5. Brandoff JF, Silber JS, Vaccaro AR. Contemporary alternatives to synthetic bone grafts for spine surgery. Am J Orthop (Belle Mead NJ). 2008;37(8):410-4.

6. Rihn JA, Patel R, Makda J, Hong J, Anderson DG, Vaccaro AR, et al. Complications associated with single-level transforaminal lumbar interbody fusion. Spine J. 2009;9(8):623-9.

7. Connolly PJ, Esses SI, Kostuik JP. Anterior cervical fusion: outcome analysis of patients fused with and without anterior cervical plates. J Spinal Disord. 1996;9(3):202-6.

8. Mroz TE, Joyce MJ, Steinmetz MP, Lieberman IH, Wang JC. Musculoskeletal allograft risks and recalls in the United States. J Am Acad Orthop Surg. 2008;16(10):559-65.

9. Mroz TE, Joyce MJ, Lieberman IH, Steinmetz MP, Benzel EC, Wang JC. The use of allograft bone in spine surgery: is it safe? Spine J. 2009 Apr;9(4):303-8.

10. Kwon B, Jenis LG. Carrier materials for spinal fusion. Spine J. 2005;5(6 Suppl):224S-230S.

11. Epstein NE. Efficacy of different bone volume expanders for augmenting lumbar fusions. Surg Neurol. 2008;69(1):16-9.

12. Buttermann GR. Prospective nonrandomized comparison of an allograft with bone morphogenic protein versus an iliac-crest autograft in anterior cervical discectomy and fusion. Spine J. 2008;8(3):426-35

13. Furlan JC, Perrin RG, Govender PV, Petrenko Y, Massicotte EM, Rampersaud YR, et al. Use of osteogenic protein-1 in patients at high risk for spinal pseudarthrosis: a prospective cohort study assessing safety, health-related quality of life, and radiographic fusion. Invited submission from the Joint Section on Disorders of the Spine and Peripheral Nerves, March 2007. J Neurosurg Spine. 2007;7(5):486-95. 
14. Ramzi N, Ribeiro-Vaz G, Fomekong E, Lecouvet FE, Raftopoulos C. Long term outcome of anterior cervical discectomy and fusion using coral grafts. Acta Neurochir (Wien). 2008;150(12):1249-56;

15. Bizette C, Raul JS, Orhan B, Jacquet G, Czorny A. Results of cervical interbody fusion with coral grafts. Neurochirurgie. 1999;45(1):4-14.

16. Lee KJ, Roper JG, Wang JC. Demineralized bone matrix and spinal arthrodesis. Spine J. $2005 ; 5(6$ Suppl):217S-223S.

17. Cammisa FP Jr, Lowery G, Garfin SR, Geisler FH, Klara PM, McGuire RA, et al. Two-year fusion rate equivalency between Grafton DBM gel and autograft in posterolateral spine fusion: a prospective controlled trial employing a side-by-side comparison in the same patient. Spine (Phila Pa 1976). 2004;29(6):660-6.

18. Girardi FP, Cammisa FP Jr. The effect of bone graft extenders to enhance the performance of iliac crest bone grafts in instrumented lumbar spine fusion. Orthopedics. 2003;26(5 Suppl):s545-8.

19. Price $\mathrm{CT}$, Connolly JF, Carantzas $\mathrm{AC}$, llyas I. Comparison of bone grafts for posterior spinal fusion in adolescent idiopathic scoliosis. Spine (Phila Pa 1976). 2003;28(8):793-8.

20. Sassard WR, Eidman DK, Gray PM, Block JE, Russo R, Russell JL, et al. Augmenting local bone with Grafton demineralized bone matrix for posterolateral lumbar spine fusion: avoiding second site autologous bone harvest. Orthopedics. 2000;23(10):1059-64.

21. Cahill KS, Chi JH, Day A, Claus EB. Prevalence, complications, and hospital charges associated with use of bone-morphogenetic proteins in spinal fusion procedures. JAMA. 2009;302(1):58-66

22. Vaidya R, Sethi A, Bartol S, Jacobson M, Coe C, Craig JG. Complications in the use of rhBMP-2 in PEEK cages for interbody spinal fusions. J Spinal Disord Tech. 2008;21(8):557-62

23. Hiremath GK, Steinmetz MP, Krishnaney AA. Is it safe to use recombinant human bone morphogenetic protein in posterior cervical fusion? Spine (Phila Pa 1976). 2009;34(9):885-9

24. Mindea SA, Shih P, Song JK. Recombinant human bone morphogenetic protein-2-induced radiculitis in elective minimally invasive transforaminal lumbar interbody fusions: a series review. Spine (Phila Pa 1976). 2009;34(14):1480-4.

25. Geibel PT, Boyd DL, Slabisak V. The use of recombinant human bone morphogenic pro- tein in posterior interbody fusions of the lumbar spine: a clinical series. J Spinal Disord Tech. 2009;22(5):315-20.

26. Acharya NK, Kumar RJ, Varma HK, Menon VK. Hydroxyapatite-bioactive glass ceramic composite as stand-alone graft substitute for posterolateral fusion of lumbar spine: a prospective, matched, and controlled study. J Spinal Disord Tech. 2008;21(2):106-11.

27. Hagel A, Zeh A, Hein W, Held A, Wohlrab D. Comparison of anterior lumbar fusion rates after circumferential fusion using beta-tricalcium phosphate (Cerasorb) versus autologous iliac crest spongiosa. Z Orthop Unfall. 2007;145(4):488-92.

28. Neen D, Noyes D, Shaw M, Gwilym S, Fairlie N, Birch N. Healos and bone marrow aspirate used for lumbar spine fusion: a case controlled study comparing healos with autograft. Spine (Phila Pa 1976). 2006;31(18):E636-40.

29. Xie Y, Chopin D, Hardouin P, Lu J. Clinical, radiological and histological study of the failure of cervical interbody fusions with bone substitutes. Eur Spine $\mathrm{J}$. 2006;15(8):1196-203

30. Carlisle EM. In vivo requirement for silicon in articular cartilage and connective tissue formation in the chick. J Nutr. 1976 :106(4):478-84.

31. Schwarz K, Milne DB. Growth-promoting effects of silicon in rats. Nature. 1972;239(5371):333-4

32. Gao T, Uludag H. Effect of molecular weight of thermoreversible polymer on in vivo retention of rhBMP-2. J Biomed Mater Res. 2001;57(1):92-100.

33. Xynos ID, Edgar AJ, Buttery LD, Hench LL, Polak JM. Gene-expression profiling of human osteoblasts following treatment with the ionic products of Bioglass $45 \mathrm{~S} 5$ dissolution. J Biomed Mater Res. 2001;55(2):151-7.

34. Reffitt DM, Ogston N, Jugdaohsingh R, Cheung HF, Evans BA, Thompson RP, et al. Orthosilicic acid stimulates collagen type 1 synthesis and osteoblastic differentiation in human osteoblast-like cells in vitro. Bone. 2003;32(2):127-35.

35. Jugdaohsingh R, Tucker KL, Qiao N, Cupples LA, Kiel DP, Powell JJ. Dietary silicon intake is positively associated with bone mineral density in men and premenopausal women of the Framingham Offspring cohort. J Bone Miner Res. 2004;19(2):297-307.

36. Bissé $E$, Epting $T$, Beil $A$, Lindinger $G$, Lang $H$, Wieland $H$. Reference values for serum silicon in adults. Anal Biochem. 2005;337(1):130-5. 